\title{
Hippocampal and Deep Gray Matter Nuclei Atrophy Is Relevant for Explaining Cognitive Impairment in MS: A Multicenter Study
}

\author{
(DD. Damjanovic, DP. Valsasina, (DM.A. Rocca, (D) M.L. Stromillo, (D)A. Gallo, DC. Enzinger, (D) H.E. Hulst, (D) A. Rovira, (D) N. Muhlert, \\ (D) N. De Stefano, (1) A. Bisecco, (D)F. Fazekas, (D).J. Arévalo, (D).A. Yousry, and (D) M. Filippi
}

\begin{abstract}
BACKGROUND AND PURPOSE: The structural MR imaging correlates of cognitive impairment in multiple sclerosis are still debated. This study assessed lesional and atrophy measures of white matter and gray matter involvement in patients with MS acquired in 7 European sites to identify the MR imaging variables most closely associated with cognitive dysfunction.
\end{abstract}

MATERIALS AND METHODS: Brain dual-echo, 3D T1-weighted, and double inversion recovery scans were acquired at 3 T from 62 patients with relapsing-remitting MS and 65 controls. Patients with at least 2 neuropsychological tests with abnormal findings were considered cognitively impaired. Focal WM and cortical lesions were identified, and volumetric measures from WM, cortical GM, the hippocampus, and deep GM nuclei were obtained. Age- and site-adjusted models were used to compare lesion and volumetric MR imaging variables between patients with MS who were cognitively impaired and cognitively preserved. A multivariate analysis identified MR imaging variables associated with cognitive scores and disability.

RESULTS: Twenty-three patients (38\%) were cognitively impaired. Compared with those with who were cognitively preserved, patients with MS with cognitive impairment had higher T2 and T1 lesion volumes and a trend toward a higher number of cortical lesions. Significant brain, cortical GM, hippocampal, deep GM nuclei, and WM atrophy was found in patients with MS with cognitive impairment versus those who were cognitively preserved. Hippocampal and deep GM nuclei atrophy were the best predictors of cognitive impairment, while WM atrophy was the best predictor of disability.

CONCLUSIONS: Hippocampal and deep GM nuclei atrophy are key factors associated with cognitive impairment in MS. These MR imaging measures could be applied in a multicenter context, with cognition as clinical outcome.

ABBREVIATIONS: $\mathrm{Cl}=$ cognitively impaired; $\mathrm{CL}=$ cortical lesion; $\mathrm{CP}=$ cognitively preserved; $\mathrm{DIR}=$ double inversion recovery; $\mathrm{EDSS}=$ Expanded Disability Status Scale; $\mathrm{HC}=$ healthy controls; $\mathrm{LV}=$ lesion volumes; $\mathrm{WCST}=$ Wisconsin Card Sorting Test

$\mathbf{C}^{\circ}$ gnitive impairment is a frequent finding in patients with multiple sclerosis, with $40 \%-70 \%$ of patients showing cognitive deficits. ${ }^{1}$ The most affected domains are attention, information-processing speed, executive functions, and memory and

Received June 8, 2016; accepted after revision August 11.

From the Neuroimaging Research Unit (D.D., P.V., M.A.R., M.F.) and Department of Neurology (M.A.R., M.F.), Institute of Experimental Neurology, Division of Neuroscience, San Raffaele Scientific Institute, Vita-Salute San Raffaele University, Milan Italy; Center for Radiology and MRI of Clinical Center of Serbia (D.D.), Faculty of Medicine, University of Belgrade, Belgrade, Serbia; Department of Neurological and Behavioural Sciences (M.L.S., N.D.S.), University of Siena, Siena, Italy; MRI Center "SUN-FISM" (A.G., A.B.), Second University of Naples and Institute of Diagnosis and Care "Hermitage-Capodimonte," Naples, Italy; I Division of Neurology (A.G., A.B.), Department of Medical, Surgical, Neurological, Metabolic and Aging Sciences, Second University of Naples, Naples, Italy; Department of Neurology (C.E., F.F.) and Division of Neuroradiology (C.E.), Vascular and Interventional Radiology, Department of Radiology, Medical University of Graz, Graz, Austria; Department of Radiology and Nuclear Medicine (H.E.H.), MS Centre Amsterdam, VU University Medical Centre, Amsterdam, Netherlands; Magnetic Resonance Unit (A.R., M.J.A.), Department of Radiology and MS Centre of Catalonia, Hospital Universitari Vall d'Hebron, Barcelona, Spain; and NMR Research Unit (N.M., T.A.Y.), Queen Square MS Centre, University College London Institute of Neurology, London, UK. visuospatial abilities. ${ }^{1}$ Given its dramatic effect on the activities of patients' daily lives, there is a critical need to define the pathophysiologic mechanisms of cognitive impairment in MS, to develop markers for its monitoring, and to identify valid therapeutic strategies.

Many studies tried to characterize the structural MR imaging correlates of cognitive impairment in patients with MS. T2 and T1 lesion volumes were found to be generally higher in patients with

This study has been partially supported by a grant from the Italian Ministry of Health (GR-2009-1529671). The MS Center Amsterdam is supported by the Dutch MS Research Foundation (grant 09-538d). Dr Damjanovic was supported by an ECTRIMS-MAGNIMS fellowship.

Please address correspondence to Massimo Filippi, MD, Neuroimaging Research Unit, Institute of Experimental Neurology, Division of Neuroscience, San Raffaele Scientific Institute, Vita-Salute San Raffaele University, Via Olgettina, 60, 20132 Milan, Italy; e-mail: filippi.massimo@hsr.it

- Indicates open access to non-subscribers at www.ajnr.org

三 Indicates article with supplemental on-line table.

http://dx.doi.org/10.3174/ajnr.A4952 
MS who were cognitively impaired (CI) than in those who were cognitively preserved (CP), ${ }^{2-5}$ and poor performance on a given neuropsychological test correlated with the presence of lesions in relevant WM tracts. ${ }^{2,4,6} \mathrm{GM}$ damage was also variously related to cognitive impairment in these patients. In particular, higher cortical lesion (CL) volume on double inversion recovery (DIR) sequences, ${ }^{7,8}$ reduced neocortical and total GM volume, ${ }^{7,9}$ and structural abnormalities within strategic GM regions, such as the thalamus, putamen, and hippocampus, were related to the presence and severity of cognitive symptoms. ${ }^{10-13}$

Despite the clear association between isolated measures of structural CNS damage (eg, WM lesion volume or GM volume) and cognitive performance, when multiparametric models were applied to identify the imaging correlates of cognitive impairment, conflicting results have been obtained, with some studies identifying a prominent contribution of WM damage ${ }^{5,14}$ and others underpinning the relevance of cortical or deep GM nuclei involvement. ${ }^{3,9,13}$ Additionally, composite models explaining cognitive impairment have only been tested at single sites, in selected groups of patients. ${ }^{13}$ Lesional and volumetric MR imaging measures of WM and GM damage might be used as outcome measures for disease-monitoring purposes, both in observational and treatment studies. However, to test the utility of these measures as objective imaging biomarkers of cognitive impairment, a validation of such multiparametric models in a multicenter setting is needed.

Here, we hypothesized that GM loss might be the most relevant contributor of cognitive impairment in MS. To test our hypothesis, we characterized the structural MR imaging correlates of cognitive impairment in a group of patients with MS acquired in 7 European sites by analyzing lesional and atrophy measures of WM and GM involvement, and we identified the set of MR imaging variables most closely associated with cognitive dysfunction.

\section{MATERIALS AND METHODS}

\section{Ethics Committee Approval}

Local ethics approval was obtained at all sites; all subjects gave written informed consent.

\section{Subjects}

Subjects were recruited from January 2009 to May 2012 as part of a project on imaging correlates of cognitive impairment in MS at 7 European centers. The results of regional analysis of GM and WM damage by using surface-based ${ }^{15}$ and voxelwise techniques ${ }^{16}$ have been previously reported.

All subjects had to be between 20 and 65 years of age. Patients had to have a diagnosis of relapsing-remitting MS, ${ }^{17}$ no relapse or corticosteroid treatment within the month before scanning, and no history of psychiatric conditions, including major depression.

The final dataset included 62 patients with MS (22/40 men/ women; mean age, $39.5 \pm 8.5$ years; mean disease duration, 8.2 years [range, 2-33 years]; median Expanded Disability Status Scale [EDSS] score, 2.0 [range, $0.0-6.0$ ]) and 65 healthy controls (HC) (27/38 men/women; mean age, $35.8 \pm 9.4$ years) (On-line Table). Sex did not differ between $\mathrm{HC}$ and patients with MS ( $P=$ $.7)$, whereas HC were younger than patients with MS $(P=.006)$.
Thus, age was included as a nuisance covariate in all statistical models.

\section{Clinical and Neuropsychological Assessment}

Within 48 hours from the MR imaging acquisition, patients with MS underwent a neurologic evaluation, with an EDSS rating and a neuropsychological assessment performed at each site by an experienced neuropsychologist unaware of the MR imaging results, using validated translations of the neuropsychological tests. Cognitive performance was assessed by using the Brief Repeatable Battery of Neuropsychological Tests, ${ }^{18}$ including the Selective Reminding Test to assess verbal memory; the 10/36 Spatial Recall Test to assess visuospatial memory; the Symbol Digit Modalities Test and the Paced Auditory Serial Addition Test 2 seconds and 3 seconds to assess attention and information processing speed; and the Word List Generation test to assess verbal fluency. As previously described, ${ }^{19} z$ scores for each of the previous domains and a global $z$ score of cognitive function (obtained by averaging $\mathrm{z}$ scores of all tests) were calculated.

In addition, the Wisconsin Card Sorting Test (WCST) was administered to evaluate executive functions. ${ }^{20}$ Performance on the WCST was evaluated by computing scores related to the total errors, the number of perseverative errors, and the number of perseverative responses. ${ }^{20}$ Patients with a score $\geq 2$ SDs below normative values in at least 1 of these measures were considered impaired on the WCST.

Patients with at least 2 abnormal test results (defined as a score $\geq 2$ SDs below the normative value provided by Boringa et $\mathrm{al}^{21}$ for the Brief Repeatable Battery of Neuropsychological Tests and by Heaton $^{20}$ for the WCST) were considered CI, as previously described. ${ }^{16}$

\section{MR Imaging Acquisition}

With 3T scanners (Centers I and VI: Signa; GE Healthcare, Milwaukee, Wisconsin; Centers II, III, and IV: Magnetom Trio; Siemens, Erlangen, Germany; Centers V and VII: Intera; Philips Healthcare, Best, the Netherlands), centers performed the following brain sequences: 1 ) dual-echo TSE: TR $=4000-5380 \mathrm{~ms}$; $\mathrm{TE}_{1}=10-23 \mathrm{~ms} ; \mathrm{TE}_{2}=90-102 \mathrm{~ms}$; echo-train length $=5-11 ; 44$ contiguous, 3-mm-thick axial sections parallel to the anterior/ posterior commissure plane; matrix $=256 \times 192$; $\mathrm{FOV}=240 \times$ $180 \mathrm{~mm}^{2}$ (rectangular FOV $\left.=75 \%\right) ; 2$ ) 3D T1-weighted scan: $\mathrm{TR}=5.5-8.3 \mathrm{~ms}$ (for GE Healthcare/Philips Healthcare scanners) or 1900-2300 ms (for Siemens scanners); TE $=1.7-3.0 \mathrm{~ms}$; flip angle $=8^{\circ}-12^{\circ} ; 176-192$ sagittal sections with thickness $=1$ $\mathrm{mm}$ and in-plane resolution $=1 \times 1 \mathrm{~mm}^{2} ; 3$ ) double inversion recovery sequence: $\mathrm{TR}=7500-16,000 \mathrm{~ms}$; $\mathrm{TE}=25-317 \mathrm{~ms} ; \mathrm{TI}_{1}$ $=325-500 \mathrm{~ms} ; \mathrm{TI}_{2}=2100-3870 \mathrm{~ms}$; echo-train length $=10-13$; 44 contiguous, 3 -mm-thick axial sections parallel to the anterior/ posterior commissure plane; matrix $=256 \times 192$; FOV $=240 \times$ $180 \mathrm{~mm}^{2}$ (rectangular FOV $=75 \%$ ), apart from Center I, which performed a $3 \mathrm{D}$ acquisition with 140 sagittal sections with thickness $=1.2 \mathrm{~mm}$; matrix $=224 \times 224 ; \mathrm{FOV}=220 \times 220 \mathrm{~mm}^{2}$.

\section{MR Imaging Analysis}

Center V performed central analysis of MR imaging scans. WM and GM lesions were identified by a radiologist (D.D., with 10 


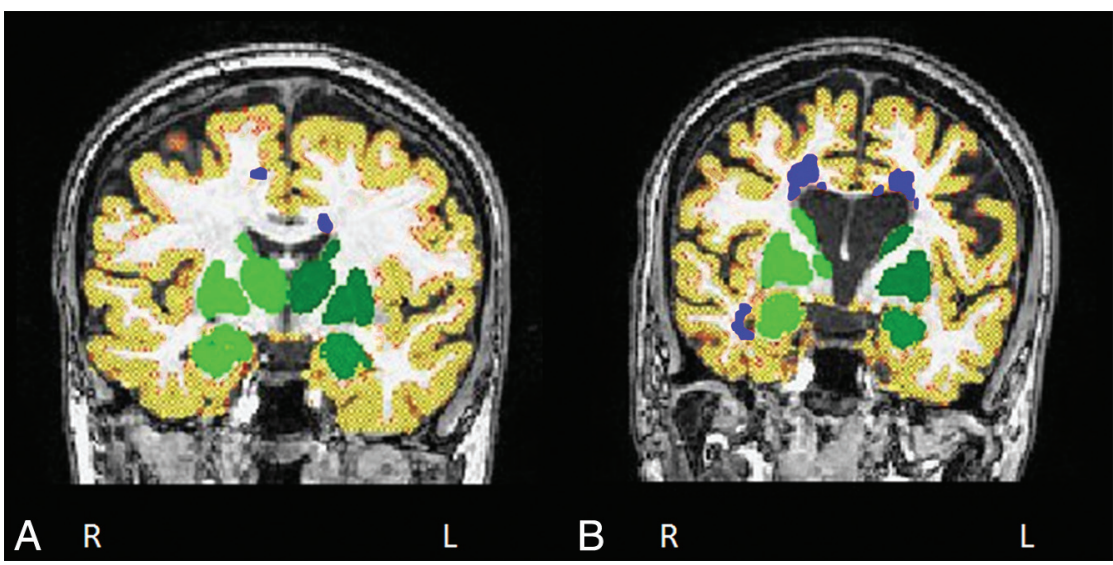

FIG 1. Illustrative examples of segmentation of cortical gray matter volume (in red-yellow), deep gray matter volume (in green), and lesions (in blue) in patients with multiple sclerosis without $(A)$ and with $(B)$ cognitive impairment. Images are in radiologic convention.

years of experience) supervised by a neurologist (M.A.R., with 20 years of experience). Brain T2-hyperintense and T1-hypointense lesion volumes (LV) were measured on dual-echo and 3D T1weighted scans, respectively, by using a local thresholding segmentation technique (Jim 6.0 software; http://www.xinapse.com/ home.php). DIR scans from Center I were first reformatted to the axial orientation and resampled to $3-\mathrm{mm}$ section thickness, to standardize evaluation of DIR lesions across sites. Then, DIR images were used to count CLs according to consensus recommendations. ${ }^{22}$ CLs included the following: 1) lesions confined to the cortical ribbon without involving the underlying subcortical WM (pure intracortical lesions), and 2) mixed WM/GM lesions (type I) with a prominent extension within the GM ( $\geq 75 \%)$. Attention was paid to the exclusion of artifacts. Pure, mixed WM/GM, and total DIR LV were calculated by using Jim software.

Normalized brain volume, normalized GM volume, normalized cortical GM volume, and normalized WM volume were measured on 3D T1-weighted scans by using FSL SIENAX (http://fsl.fmrib.ox.ac.uk/fsl/fslwiki/SIENA) after refilling of T1-hypointense lesions. ${ }^{23}$ Automatic segmentation of the thalamus, caudate, putamen, pallidum, hippocampus, amygdala, and accumbens was performed on 3D T1-weighted scans by using the FMRIB Integrated Registration and Segmentation Tool (FIRST; http://fsl.fmrib.ox.ac.uk/fsl/fslwiki/FIRST) software (Fig 1). ${ }^{24}$ The volume of these structures was multiplied by the head-normalization factor derived from SIENAX.

\section{Statistical Analysis}

Demographic and clinical characteristics, together with lesional and volumetric MR imaging variables, were reported as means and ranges, or frequencies and percentages, for continuous and categoric variables, respectively. Brain T2 and T1 LV were logtransformed due to their skewed distribution. Given the similar behavior of right and left deep GM nuclei, deep GM volumes were averaged across hemispheres before statistical analysis. This process avoided multicollinearity and reduced the number of comparisons. The normalized volume of deep GM nuclei (ie, the sum of the thalamus, caudate, putamen, pallidum, amygdala, and accumbens) was also obtained. Between-center heterogeneity of
MR imaging variables was tested by using ANOVA models for continuous variables and Pearson $\chi^{2}$ tests for categoric variables (apart from CL numbers, which were entered into negative binomial models).

Comparisons between $\mathrm{HC}$ and patients with MS and between $\mathrm{HC}$ and patients with MS who were $\mathrm{CP}$ and $\mathrm{CI}$ of lesional and volumetric MR imaging quantities were performed by using ageadjusted generalized linear random-effect models, accounting for clustering (ie, subjects within the recruitment site) by using random intercepts and an unstructured covariance matrix.

Univariate correlations between clinical, neuropsychological, and lesion/volumetric MR imaging variables were also assessed by using generalized linear random-effects models, accounting for clustering by using random intercepts and an unstructured covariance matrix, adjusting for age. The effect size of correlations was reported by using standardized regression coefficients. The same modeling strategy was used to identify MR imaging variables independently associated with cognitive and EDSS scores by using a stepwise variable selection. Variables were selected by using a significance level of .10 for entry into the model and a significance level of .05 to remain in the multivariate model.

A $P$ value $<.05$ was considered statistically significant (SAS Release 9.1.3 software; SAS Institute, Cary, North Carolina). All results were adjusted for multiple comparisons by using the false discovery rate approach. ${ }^{25}$

\section{RESULTS}

Demographic, Clinical, and Neuropsychological Measures The On-line Table summarizes the main demographic and clinical characteristics of the study subjects. No site heterogeneity was found for sex and disease duration, while age and EDSS were heterogeneous among sites (On-line Table).

Twenty-three (37\%) patients with MS were CI. The domains most frequently involved were the following: attention and information-processing speed (43\%), verbal memory (27\%), spatial memory (21\%), and fluency (16\%). All patients impaired on the WCST $(n=15)$ were also classified as CI on the Brief Repeatable Battery of Neuropsychological Tests. Compared with CP patients, those with MS who were CI were significantly older $(P=.007)$, whereas no difference was found for $\operatorname{sex}(P=.2)$, EDSS score $(P=$ $.3)$, disease duration $(P=.4)$, and education (mean years of education, $13.7 \pm 3.1$ years in CP MS and $13.5 \pm 3.4$ years in $\mathrm{CI}$ MS, $P=.8$ ).

\section{Lesion Findings}

There was no significant between-site heterogeneity for T2 $(P=$ $.17)$, T1 $(P=.22)$, and DIR $(P=.09) \mathrm{LV}$ (Table 1$)$. The number of CLs was significantly heterogeneous among sites $(P<.001$ for total and pure CLs, $P=.07$ for mixed CLs). Compared with CP 
Table 1: Lesion and volumetric MRI findings in healthy controls and patients with MS, first considered as a whole and then divided into patients who are cognitively preserved and cognitively impaired

\begin{tabular}{|c|c|c|c|c|c|c|}
\hline & $\mathrm{HC}$ & MS & $P^{a}$ & $\begin{array}{c}\text { Cognitively Preserved } \\
\text { Patients }\end{array}$ & $\begin{array}{c}\text { Cognitively Impaired } \\
\text { Patients }\end{array}$ & $P^{a}$ \\
\hline $\mathrm{T} 2 \mathrm{LV}(\mathrm{mL})(\mathrm{SD})$ & NA & $10.6(13.9)$ & NA & $7.1(9.6)$ & $16.5(17.8)$ & .01 \\
\hline TT LV (mL) (SD) & NA & $5.5(5.8)$ & NA & $3.6(3.5)$ & $8.7(7.6)$ & .01 \\
\hline DIR LV (mL) (SD) & NA & $0.4(0.7)$ & NA & $0.4(0.9)$ & $0.4(0.4)$ & .4 \\
\hline Median CL (No.) (range) & NA & $3(0-33)$ & NA & $2.0(0-33)$ & $6.0(1-16)$ & $.5^{\mathrm{b}}$ \\
\hline Median pure CL (No.) (range) & NA & $1(0-14)$ & NA & $0.5(0-14)$ & $1.5(0-11)$ & $.8^{\mathrm{b}}$ \\
\hline Median mixed CL (No.) (range) & NA & $2(0-19)$ & NA & $1.0(0-19)$ & $4.0(0-9)$ & $.9^{\mathrm{b}}$ \\
\hline $\mathrm{NBV}(\mathrm{mL})(\mathrm{SD})$ & $1533(79)$ & $1436(145)$ & $<.001$ & $1460(98)$ & 1395 (1113) & .006 \\
\hline $\mathrm{NGMV}(\mathrm{mL})(\mathrm{SD})$ & $826(56)$ & $776(67)$ & .0002 & $793(68)$ & $748(57)$ & .02 \\
\hline NCGMV (mL) (SD) & $638(48)$ & $599(53)$ & .0004 & $612(55)$ & $578(43)$ & .02 \\
\hline $\mathrm{NWMV}(\mathrm{mL})(\mathrm{SD})$ & $706(40)$ & $659(68)$ & $<.001$ & $667(54)$ & $645(88)$ & .03 \\
\hline Total normalized deep GM volume (mL) (SD) & $28.1(1.7)$ & $24.7(3.1)$ & $<.001$ & $25.8(2.4)$ & $23.0(3.5)$ & $<.001$ \\
\hline Average normalized thalamus volume (mL) (SD) & $11.1(0.7)$ & $9.7(1.3)$ & $<.001$ & $10.1(1.0)$ & $9.0(1.4)$ & .0007 \\
\hline Average normalized caudate volume (mL) (SD) & $5.0(0.5)$ & $4.4(0.7)$ & $<.001$ & $4.5(0.6)$ & $4.1(0.7)$ & .02 \\
\hline Average normalized putamen volume (mL) (SD) & $6.8(0.6)$ & $6.1(0.9)$ & $<.001$ & $6.5(0.7)$ & $5.5(0.9)$ & $<.001$ \\
\hline Average normalized pallidum volume (mL) (SD) & $2.5(0.3)$ & $2.2(0.2)$ & $<.001$ & $2.3(0.1)$ & $2.1(0.3)$ & .05 \\
\hline Average normalized hippocampus volume (mL) (SD) & $5.3(0.4)$ & $4.8(0.6)$ & $<.001$ & $5.0(0.5)$ & $4.5(0.7)$ & .004 \\
\hline Average normalized amygdala volume (mL) (SD) & $1.8(0.3)$ & $1.6(0.3)$ & .001 & $1.7(0.3)$ & $1.5(0.3)$ & .05 \\
\hline Average normalized accumbens volume (mL) (SD) & $0.7(0.1)$ & $0.6(0.1)$ & .004 & $0.6(0.1)$ & $0.5(0.1)$ & .01 \\
\hline
\end{tabular}

Note:-NA indicates not applicable; NBV, normalized brain volume; NGMV, normalized GM volume; NCGMV, normalized cortical GM volume; NWMV, normalized WM volume.

a Age- and site-adjusted linear mixed-effect model with random intercept for recruitment site.

${ }^{\mathrm{b}}$ Age-adjusted negative binomial mixed-effect model with random intercept for recruitment site (false discovery rate-corrected).

Table 2: Correlations between lesion and volumetric MRI measures of WM and GM with clinical and neuropsychological variables (ageadjusted linear mixed-effect model with random intercept for recruitment site, false discovery rate-corrected) ${ }^{\mathrm{a}}$

\begin{tabular}{|c|c|c|c|c|c|}
\hline MRI Measure & $\begin{array}{c}\text { Global Cognitive } \\
\text { Z Score }\end{array}$ & $\begin{array}{l}\text { Attention } \\
\text { Z Score }\end{array}$ & $\begin{array}{c}\text { Visual Memory } \\
\text { Z Score }\end{array}$ & $\begin{array}{l}\text { Executive Functions } \\
\text { (WCSTpr) }\end{array}$ & EDSS \\
\hline T2 LV & $-0.35(.01)$ & $-0.33(.008)$ & $-0.34(.03)$ & $-0.25(.06)$ & NS \\
\hline TT LV & $-0.31(.02)$ & $-0.31(.01)$ & $-0.33(.03)$ & $-0.28(.05)$ & NS \\
\hline NBV & $0.44(.01)$ & $0.44(.001)$ & $0.35(.03)$ & NS & $-0.33(.01)$ \\
\hline NGMV & $0.36(.01)$ & $0.31(.01)$ & NS & NS & NS \\
\hline NCGMV & $0.42(.01)$ & $0.36(.01)$ & NS & NS & NS \\
\hline NWMV & $0.37(.01)$ & $0.40(.004)$ & $0.35(.03)$ & NS & $-0.35(.008)$ \\
\hline Total normalized deep GM volume & $0.40(.001)$ & $0.47(<.001)$ & $0.33(.04)$ & NS & $-0.29(.03)$ \\
\hline Average normalized thalamus volume & $0.36(.002)$ & $0.41(.0005)$ & $0.30(.05)$ & NS & NS \\
\hline Average normalized caudate volume & $0.40(.001)$ & $0.50(<.001)$ & NS & NS & NS \\
\hline Average normalized putamen volume & $0.40(.001)$ & $0.46(.0001)$ & $0.37(.03)$ & NS & $-0.30(.03)$ \\
\hline Average normalized pallidum volume & $0.25(.03)$ & $0.34(.003)$ & NS & NS & $-0.35(.01)$ \\
\hline Average normalized hippocampus volume & $0.39(.001)$ & $0.34(.003)$ & NS & NS & NS \\
\hline Average normalized amygdala volume & $0.33(.01)$ & $0.33(.01)$ & NS & NS & NS \\
\hline Average normalized accumbens volume & $0.32(.009)$ & $0.36(.003)$ & NS & NS & NS \\
\hline
\end{tabular}

Note:-NS indicates not significant; WCSTpr, Wisconsin Card Sorting Test, number of perseverative responses; NBV, normalized brain volume; NGMV, normalized GM volume; NCGMV, normalized cortical GM volume; NWMV, normalized WM volume.

${ }^{a}$ Correlations are reported as standardized coefficients (to include size effect), and $P$ values are reported in parentheses.

patients, those with MS who were CI had higher T2 and T1 LV, whereas DIR LV and the number of CLs did not differ between groups (Table 1).

\section{Volumetric MR Imaging Findings}

Normalized brain volume, normalized GM volume, normalized cortical GM volume, and normalized white matter volume were significantly heterogeneous across sites $(P=<.001-.04)$, while this was not the case in deep GM nuclei volumes, apart from the amygdala $(P<.001)$. Volumes of deep GM nuclei were similar to those obtained in previous studies. ${ }^{26,27}$ All structures were atrophied in patients with MS compared with $\mathrm{HC}(P<.001)$ and in patients with MS who were CI versus CP (Table 1).

\section{Analysis of Correlation}

T2 LV and T1 LV were significantly correlated with global and partial cognitive scores, but not with EDSS (Table 2). Lower nor- malized brain volume was associated with worse neuropsychological scores and higher disability. When looking at brain tissue compartments (ie, WM and GM), we found that EDSS was correlated with normalized white matter volume, whereas the highest correlation with the global cognitive score was found with decreased normalized cortical GM volume (Table 2). Decreased volumes of all deep GM nuclei and the hippocampus were significantly associated with global and attention neuropsychological scores. Lower putamen and pallidum volumes were correlated with higher EDSS scores. There were no significant correlations between MR imaging measures and disease duration, verbal memory, or fluency $z$ scores. Furthermore, no correlation was found between CLs and clinical/neuropsychological measures.

The multivariate analysis retained average hippocampal volume (explained variance $=15 \%, P=.0002$ ) as the best predictor of global cognitive $z$ scores, normalized volume of deep GM nuclei (explained variance $=19 \%, P<.001)$ as the best predictor of 
attention $z$ scores, normalized brain volume (explained variance $=10 \%, P=.006)$ as the best predictor of visual memory $z$ scores, and normalized WM volume (explained variance $=8 \%$, $P=.008)$ as the best predictor of EDSS scores.

\section{DISCUSSION}

This is one of the first multicenter studies characterizing lesion and volumetric MR imaging correlates of cognitive impairment in patients with MS, to our knowledge. More severe damage of both WM and GM compartments was found in patients with MS who were CI compared with CP, despite similar levels of clinical disability. WM lesion volume and GM atrophy were both correlated with the severity of cognitive deficits, supporting the notion that different substrates contribute to cognitive dysfunction. However, on multivariate analysis, measures of GM volume were identified as the best correlate of global cognitive impairment. Overall, our results suggest that volumes of the hippocampus and deep GM nuclei may be reliable biomarkers of cognitive impairment in MS and might be used in multicenter observational or treatment studies.

In agreement with the results of previous studies, ${ }^{2-4,18}$ we found that both T2 and T1 LV were higher in patients with MS who were CI than CP. This finding seems to indicate that disconnection, caused by focal WM damage, may represent one of the factors contributing to the development of cognitive impairment in MS. ${ }^{4}$ Hyperintensities on T2-weighted MR imaging have a relatively low pathologic specificity because they relate to a variety of processes, while $\mathrm{T} 1$ hypointensities seem to be more closely associated with severe demyelination and axonal loss. ${ }^{28}$ The significantly higher T1 LV we found in CI versus CP MS suggest that not only the extent but also the severity of damage within MS lesions might be relevant in determining cognitive decline in these patients.

Contrary to our expectations, we found only a nonsignificant trend toward a higher number of CLs in patients with MS who were CI compared with CP. This is in contrast to results of previous studies, ${ }^{7,8}$ which reported associations between the number and volume of CLs and cognitive impairment. At least 2 factors could help explain this discrepancy. First, DIR sequences can be challenging to standardize across scanners, and in this study, they were acquired with different geometry (3D versus $2 \mathrm{D})$ and parameters across sites. These differences may have contributed to the heterogeneity of CL counts and volumes seen across sites. In comparison, dual-echo and 3D T1-weighted scans were acquired with a relatively standardized protocol, and T2 and T1 LV were accordingly similar among sites. Second, DIR sequences are more prone to artifacts than T2- and T1-weighted sequences, which can impact the detection of CLs. ${ }^{22}$

GM atrophy can also contribute to cognitive symptoms in MS. In line with previous studies, we found significantly reduced cortical, hippocampal, and deep GM volumes in patients with MS who were CI compared with CP. Reduced neocortical volume $^{2,3,7,9}$ and a widespread pattern of regional GM atrophy ${ }^{15,16,29}$ have been frequently associated with cognitive impairment in MS, as also shown by a previous investigation of the same patient cohort. ${ }^{16} \mathrm{GM}$ pathology is known to be substantially present in MS from the earliest stages of the disease and is strongly associated with CI not only in cross-sectional but also in longitudinal studies. ${ }^{30} \mathrm{GM}$ pathology has traditionally been thought to occur secondary to injury from focal WM lesions (so-called Wallerian degeneration), but recent studies have convincingly shown that cortical inflammatory pathology from subpial demyelination also plays a critical role. ${ }^{31,32}$

Another interesting result was the widespread volume loss we found in CI compared with CP MS in deep GM nuclei and the hippocampus. This finding confirms a previous voxelwise investigation of the same cohort, ${ }^{16}$ which showed that hippocampal atrophy was among the best MR imaging variables discriminating patients with MS who were CI from those who were CP. The thalamus, hippocampus, and striatal structures represent convergence points across multiple cortical, limbic, brain stem, and cerebellar systems and have a key role for efficient information processing. ${ }^{33}$ Because a decrease in information-processing speed is one of the main features of cognitive impairment in $\mathrm{MS},{ }^{34}$ damage to these structures has frequently been linked to cognitive impairment. ${ }^{10,11,13}$ Remarkably, even if we found a significant correlation between thalamic atrophy and CI, our multivariate model indicated a major role of the hippocampus in explaining cognitive deficits. This finding partially conflicts with findings in previous studies. ${ }^{10,11}$ However, these studies did not perform a direct comparison of the relative contribution of thalamic and hippocampal atrophy with cognitive impairment. On the other hand, hippocampal injury has been associated not only with memory deficits ${ }^{35,36}$ but also with impaired visuospatial or verbal memory abilities. ${ }^{12,36}$

Our results indicated that most cognitive scores were correlated with higher $\mathrm{T} 2$ and T1 LV. This result was not the case for the EDSS, which did not correlate with lesion extent in univariate models. Moreover, univariate correlations showed that cognitive and clinical disability scores were associated with decreased global and deep GM volumes. Most interesting, multivariate correlations showed that cognitive scores were best predicted by hippocampal and deep GM loss, whereas WM loss was the most significant contributor to the EDSS score. Although we cannot exclude GM volume loss of critical cortical regions (eg, the motor cortex) also contributing to the EDSS score, the high relevance to clinical disability from normalized WM volume suggests that demyelination and the consequent disruption of WM pathways play an important role in explaining clinical (and in particular, locomotor) disability. Conversely, cortical, hippocampal, and deep GM nuclei atrophy in patients with MS was more closely associated with cognitive scores than WM atrophy. This result is in line with several previous studies, ${ }^{2,3,9}$ including a previous voxelwise investigation of the same cohort. ${ }^{16}$ This similarity suggests that the findings hold across a wide spectrum of the MS population. Moreover, because normalized cortical GM volume and normalized volume of deep GM nuclei predict cognitive deterioration with time, ${ }^{27,37}$ they might be used as reliable outcome measures in multicenter observational or treatment studies of cognitive impairment in MS.

Our study has limitations. First, the DIR acquisition protocol was not fully standardized across sites. Second, multicenter, longitudinal observations, including larger cohorts of patients with MS at different stages of the disease, are needed to validate our 
findings and to define how the development of GM and WM damage contributes to the evolution of cognitive impairment with time. Third, clinical disability was measured by using the EDSS, which is strongly weighted toward locomotor dysfunction. Composite scores (such as the Multiple Sclerosis Functional Composite) might have provided a more comprehensive evaluation of clinical impairment. Finally, we limited our analysis to a subset of possible MR imaging variables (lesions and atrophy of whole-tissue compartments), which are likely to be sensitive to only some of the pathologic substrates of the disease. Further studies should assess the added contribution of microstructural WM damage or intrinsic damage within lesions, for example, by diffusion tensor imaging or other quantitative MR imaging techniques.

\section{CONCLUSIONS}

Our study shows that GM atrophy is critical for explaining cognitive impairment in MS. If an adequate standardization of MR imaging acquisition and analysis is performed, lesional and atrophy measures of GM and WM might be used as biomarkers in future multicenter observational and treatment studies of cognitive impairment in MS.

\section{ACKNOWLEDGMENTS}

The authors are grateful to Dr Massimiliano Copetti for assisting in the statistical analysis.

Disclosures: Dusan Damjanovic-RELATED: Dr Damjanovic was supported by an ECTRIMS-MAGNIMS fellowship. Paola Valsasina-UNRELATED: Payment for Lectures including service on Speakers Bureaus: Excellence in Medical Education. Maria A. Rocca—RELATED: Grant: This study has been partially supported by a grant from the Italian Ministry of Health (GR-2009-1529671). UNRELATED: Payment for Lectures including service on Speakers Bureaus: Biogen, Novartis, Teva, and Genzyme. Antonio Gallo-UNRELATED: Payment for Lectures including service on Speakers Bureaus: Biogen, Teva, Merck Serono, Novartis, Genzyme. Hanneke E. Hulst-UNRELATED: Consultancy: Merck Serono, Genzyme, Novartis*; Grants/ Grants Pending: Dutch MS Research Foundations*; Payment for Lectures including service on Speakers Bureaus: Merck Serono, Genzyme, Roche*. Alex Rovira-UNRELATED: Consultancy: Novartis, Genzyme, Olea, Biogen; Payment for Lectures including service on Speakers Bureaus: Novartis, Teva, Stendhal, Bayer, Bracco, Genzyme, Biogen; Payment for Development of Educational Presentations: Teva, Biogen, Stendhal, Genzyme. Nicola De Stefano-UNRELATED: Consultancy: Schering, Biogen, Teva, Novartis, Genzyme, Roche, and Merck Serono; Grants/Grants Pending: Italian MS Society*; Payment for Lectures including service on Speakers Bureaus: Schering, Biogen, Teva, Novartis, Genzyme, Roche, and Merck Serono; Travel/ Accommodations/Meeting Expenses Unrelated to Activities Listed: Schering, Biogen, Teva, Novartis, Genzyme, Roche, and Merck Serono. Tarek A. YousryUNRELATED: Board Membership: Biogen*; Consultancy: IXICO*; Grants/Grants Pending: GlaxoSmithKline, Biogen, Novartis*; Payment for Lectures including service on Speakers Bureaus: Novartis. Massimo Filippi-RELATED: Grant: This study has been partially supported by a grant from the Italian Ministry of Health (GR-20091529671); UNRELATED: Board Membership: Teva; Consultancy: Biogen, Excellence in Medical Education, Novartis, and Teva; Grants/Grants Pending: Biogen, Teva, Novartis*; Payment for Lectures including service on Speakers Bureaus: Biogen, Excellence in Medical Education, Novartis, and Teva. *Money paid to the institution.

\section{REFERENCES}

1. Chiaravalloti ND, DeLuca J. Cognitive impairment in multiple sclerosis. Lancet Neurol 2008;7:1139-51 CrossRef Medline

2. Lazeron RH, Boringa JB, Schouten $\mathrm{M}$, et al. Brain atrophy and lesion load as explaining parameters for cognitive impairment in multiple sclerosis. Mult Scler 2005;11:524-31 CrossRef Medline

3. Benedict RH, Bruce JM, Dwyer MG, et al. Neocortical atrophy, third ventricular width, and cognitive dysfunction in multiple sclerosis. Arch Neurol 2006;63:1301-06 CrossRef Medline
4. Rossi F, Giorgio A, Battaglini M, et al. Relevance of brain lesion location to cognition in relapsing multiple sclerosis. PLoS One 2012; 7:e44826 CrossRef Medline

5. Hulst HE, Steenwijk MD, Versteeg A, et al. Cognitive impairment in MS: impact of white matter integrity, gray matter volume, and lesions. Neurology 2013;80:1025-32 CrossRef Medline

6. Mesaros S, Rocca MA, Kacar K, et al. Diffusion tensor MRI tractography and cognitive impairment in multiple sclerosis. Neurology 2012;78:969-75 CrossRef Medline

7. Calabrese M, Agosta F, Rinaldi F, et al. Cortical lesions and atrophy associated with cognitive impairment in relapsing-remitting multiple sclerosis. Arch Neurol 2009;66:1144-50 Medline

8. Roosendaal SD, Moraal B, Pouwels PJ, et al. Accumulation of cortical lesions in MS: relation with cognitive impairment. Mult Scler 2009; 15:708-14 CrossRef Medline

9. Amato MP, Bartolozzi ML, Zipoli V, et al. Neocortical volume decrease in relapsing-remitting MS patients with mild cognitive impairment. Neurology 2004;63:89-93 CrossRef Medline

10. Schoonheim MM, Hulst HE, Brandt RB, et al. Thalamus structure and function determine severity of cognitive impairment in multiple sclerosis. Neurology 2015;84:776-83 CrossRef Medline

11. Bisecco A, Rocca MA, Pagani E, et al; MAGNIMS Network. Connectivity-based parcellation of the thalamus in multiple sclerosis and its implications for cognitive impairment: a multicenter study. Hum Brain Mapp 2015;36:2809-25 CrossRef Medline

12. Longoni G, Rocca MA, Pagani E, et al. Deficits in memory and visuospatial learning correlate with regional hippocampal atrophy in MS. Brain Struct Funct 2015;220:435-44 CrossRef Medline

13. Daams M, Steenwijk MD, Schoonheim MM, et al. Multi-parametric structural magnetic resonance imaging in relation to cognitive dysfunction in long-standing multiple sclerosis. Mult Scler 2016;22: 608-19 CrossRef Medline

14. Sanfilipo MP, Benedict RH, Weinstock-Guttman B, et al. Gray and white matter brain atrophy and neuropsychological impairment in multiple sclerosis. Neurology 2006;66:685-92 CrossRef Medline

15. Tillema JM, Hulst HE, Rocca MA, et al; MAGNIMS Study Group. Regional cortical thinning in multiple sclerosis and its relation with cognitive impairment: a multicenter study. Mult Scler 2016;22: 901-09 CrossRef Medline

16. Preziosa P, Rocca MA, Pagani E, et al; MAGNIMS Study Group. Structural MRI correlates of cognitive impairment in patients with multiple sclerosis: a multicenter study. Hum Brain Mapp 2016;37: 1627-44 CrossRef Medline

17. Polman CH, Reingold SC, Banwell B, et al. Diagnostic criteria for multiple sclerosis: 2010 revisions to the McDonald criteria. Ann Neurol 2011;69:292-302 CrossRef Medline

18. Rao SM, Leo GJ, Haughton VM, et al. Correlation of magnetic resonance imaging with neuropsychological testing in multiple sclerosis. Neurology 1989;39:161-66 CrossRef Medline

19. Sepulcre J, Vanotti S, Hernández R, et al. Cognitive impairment in patients with multiple sclerosis using the Brief Repeatable BatteryNeuropsychology Test. Mult Scler 2006;12:187-95 CrossRef Medline

20. Heaton RK, Chelune GJ, Talley JL, et al. Wisconsin Card Sorting Test Manual: Revised and Expanded. Odessa, Florida: Psychological Assessment Resources; 1993

21. Boringa JB, Lazeron RH, Reuling IE, et al. The Brief Repeatable Battery of Neuropsychological Tests: normative values allow application in multiple sclerosis clinical practice. Mult Scler 2001;7:263-67 CrossRef Medline

22. Geurts JJ, Roosendaal SD, Calabrese M, et al; MAGNIMS Study Group. Consensus recommendations for MS cortical lesion scoring using double inversion recovery MRI. Neurology 2011;76:418-24 CrossRef Medline

23. Chard DT, Jackson JS, Miller DH, et al. Reducing the impact of white matter lesions on automated measures of brain gray and white matter volumes. J Magn Reson Imaging 2010;32:223-28 CrossRef Medline

24. Patenaude B, Smith SM, Kennedy DN, et al. A Bayesian model of 
shape and appearance for subcortical brain segmentation. Neuroimage 2011;56:907-22 CrossRef Medline

25. Benjamini Y, Hochberg Y. Controlling the false discovery rate: a practical and powerful approach to multiple testing. J R Stat Soc Ser B 1995;57:289-300

26. Debernard L, Melzer TR, Alla S, et al. Deep grey matter MRI abnormalities and cognitive function in relapsing-remitting multiple sclerosis. Psychiatry Res 2015;234:352-61 CrossRef Medline

27. Uddin MN, Lebel RM, Seres $P$, et al. Spin echo transverse relaxation and atrophy in multiple sclerosis deep gray matter: a two-year longitudinal study. Mult Scler 2016;22:1133-43 CrossRef Medline

28. van Waesberghe JH, Kamphorst W, De Groot CJ, et al. Axonal loss in multiple sclerosis lesions: magnetic resonance imaging insights into substrates of disability. Ann Neurol 1999;46:747-54 CrossRef Medline

29. Riccitelli G, Rocca MA, Pagani E, et al. Cognitive impairment in multiple sclerosis is associated to different patterns of gray matter atrophy according to clinical phenotype. Hum Brain Mapp 2011;32: 1535-43 CrossRef Medline

30. Rocca MA, Amato MP, De Stefano N, et al; MAGNIMS Study Group. Clinical and imaging assessment of cognitive dysfunction in multiple sclerosis. Lancet Neurol 2015;14:302-17 CrossRef Medline
31. Louapre C, Govindarajan ST, Gianni C, et al. Beyond focal cortical lesions in MS: an in vivo quantitative and spatial imaging study at 7T. Neurology 2015;85:1702-09 CrossRef Medline

32. Mainero C, Louapre C. Meningeal inflammation in multiple sclerosis: the key to the origin of cortical lesions? Neurology 2015;85: 12-13 CrossRef Medline

33. Leyden J, Kleinig $\mathrm{T}$. The role of the basal ganglia in data processing. Med Hypotheses 2008;71:61-64 CrossRef Medline

34. Denney DR, Lynch SG, Parmenter BA, et al. Cognitive impairment in relapsing and primary progressive multiple sclerosis: mostly a matter of speed. J Int Neuropsychol Soc 2004;10:948-56 Medline

35. Sicotte NL, Kern KC, Giesser BS, et al. Regional hippocampal atrophy in multiple sclerosis. Brain 2008;131:1134-41 CrossRef Medline

36. Sacco R, Bisecco A, Corbo D, et al. Cognitive impairment and memory disorders in relapsing-remitting multiple sclerosis: the role of white matter, gray matter and hippocampus. J Neurol 2015;262: 1691-97 CrossRef Medline

37. Amato MP, Portaccio E, Goretti B, et al. Association of neocortical volume changes with cognitive deterioration in relapsing-remitting multiple sclerosis. Arch Neurol 2007;64:1157-61 CrossRef Medline 